\title{
HIV pre-exposure prophylaxis
}

\author{
Knowledge and attitudes among \\ general practitioners
}

William Lane, Clare Heal, Jennifer Banks

\section{Background and objective \\ Human immunodeficiency virus (HIV) pre-exposure prophylaxis (PrEP) was accepted on the Australian Pharmaceutical Benefits Scheme on 1 April 2018, allowing general practitioners (GPs) to prescribe to patients in general practice. This study provides a timely assessment of GP knowledge and attitudes towards HIV PrEP.}

\section{Methods}

An explanatory mixed method approach was used. A contentvalidated questionnaire was distributed to GPs in the Mackay region to assess knowledge and attitudes towards PrEP, followed by individual in-depth interviews.

\section{Results}

Forty-five of 109 GPs (41.1\%) responded. One-third (15 of 45) had previously heard of PrEP; $71.4 \%$ (30 of 42) indicated a lack of comfort with its prescription while $60 \%$ (27 of 45 ) reported that provision of formalised guidelines was likely to increase prescription confidence. Ten GPs were interviewed until data saturation was achieved. Results supported the quantitative findings.

\section{Discussion}

GPs expressed positive attitudes towards HIV PrEP. Limited knowledge could be overcome through formalised guidelines and education.
PRE-EXPOSURE PROPHYLAXIS (PrEP) is an effective prevention against human immunodeficiency virus (HIV) ${ }^{1}$ approved by the US Food and Drug Administration in 2012 and Australia's Therapeutic Goods Administration in 2016. Prophylaxis with emcitritabine/tenofovir disoproxil fumarate is recommended for high-risk individuals as part of an overall HIV preventive care strategy. ${ }^{2,3}$

In 2010, the iPrEX study showed a $44 \%$ overall reduction in transmission rates, with a $92 \%$ reduction rate likely with full adherence. ${ }^{4} \mathrm{~A}$ number of studies were conducted in the USA and in South America both prior to and after the iPrEX trial, investigating practitioner knowledge, confidence and concerns about prescribing PrEP. ${ }^{5-10}$ Results showed varying levels of confidence in the prescription of PrEP and highlighted a desire among clinicians for further education and the provision of prescribing guidelines. ${ }^{5,9}$ However, there is a lack of equivalent research in the Australian context. Recent research has shown increased rates of rectal chlamydia and gonorrhoea in PrEP users, ${ }^{11-14}$ which in part may be attributed to 'therapeutic optimisation', the theoretical increase in high-risk behaviours by PrEP users. ${ }^{15}$ This potential risk highlights the need for clinicians to be confident in comprehensively counselling those seeking access to PrEP.

In Australia, PrEP was added to the Pharmaceutical Benefits Scheme (PBS) in April 2018, resulting in reduced prescription costs. ${ }^{16}$ Previously, PrEP was prescribed privately at a cost of up to $\$ 10,000$ per year, or accessed through clinical trials. ${ }^{17,18}$ Given the increased accessibility of PrEP, a study of Australian general practitioners (GPs) is timely. The aim of this study was to assess GPs' knowledge of and attitudes towards HIV PrEP.

\section{Methods}

This was an explanatory sequential study. ${ }^{19}$ A survey was distributed to GPs within the Mackay region, followed by in-depth semi-structured interviews to further explore GP knowledge and attitudes. Our survey was conducted prior to PBS approval and the release of Australasian Society for HIV, Viral Hepatitis and Sexual Health Medicine (ASHM) PrEP guidelines, when the majority of GPs had minimal exposure to and knowledge of this area. ${ }^{17}$

\section{Population and sample}

Mackay is a rural centre in central Queensland with a population of approximately 125,000 people. A database was established through contact with the North Queensland Primary Health Network. ${ }^{20}$ A list of practising GPs was compiled and validated with telephone confirmation. GPs on leave at the time of the survey, those who did not work in mainstream general practice (doctors based solely in skin cancer clinics) and GPs outside a radius of $30 \mathrm{~km}$ from Mackay were excluded. A database of 109 GPs was established. 


\section{Procedure}

A three-page questionnaire was developed by the principal author in consultation with co-authors. Current literature was used to inform questionnaire development and ensure content validity regarding GP knowledge of PrEP, ${ }^{5,9}$ confidence ${ }^{5,10}$ and concerns about prescribing. ${ }^{5,7,10} \mathrm{~A}$ five-point Likert scale was used to rate levels of knowledge and attitudes towards PrEP, which was consistent with scales used in previous studies. ${ }^{7}$ It was piloted by five GPs and revised accordingly for clarity. It was posted to individual GPs in two rounds - July and October 2017 - with a paid return envelope.

An invitation was further sent to all GPs on the database to participate in a qualitative interview. In-depth interviews were conducted using a semi-structured interview guide, created by the principal investigator. Questions were developed through a literature search of similar qualitative methodology with content validity ensured by study co-authors. Interviews were conducted by the principal investigator at individual GP practices and each lasted between 15 and 20 minutes. Each interview was audio-recorded and transcribed verbatim with non-verbal cues noted.

\section{Analysis}

SPSS for Windows (version 24) was used to analyse quantitative data and generate descriptive statistics and frequency tabulations. Inferential statistics were calculated using chi-square, Student $t$ test and ANOVA. Explanatory qualitative analysis followed an iterative process. ${ }^{19}$ NVIVO version 11 for Windows was used to analyse qualitative data and identify underlying themes and issues arising from the interviews.

\section{Ethics}

Queensland Health and James Cook University reciprocal ethics approval (HREC/17/QTHS/16) was granted.

\section{Results}

\section{Demographics}

The survey response rate was $41.1 \%$ (45 of 109). Participating GPs were younger (median age 44 [interquartile range (IQR)] $34,55.5)$ and more predominantly female (female:male 23:22) than expected for Australian GPs (age 45-54 years, 42.7\% female). ${ }^{17}$ There was a median of 10 -years' experience (IQR 1, 27).

\section{Knowledge}

Of the 45 GPs who responded to the questionnaire, one-third ( $\mathrm{n}=15,33.3 \%)$ had 'never heard of HIV PrEP', 51.1\% ( $\mathrm{n}=23$ ) knew 'a little' about PrEP, three (6.7\%) had 'read studies on PrEP' and only one $(2.2 \%)$ had 'previously prescribed PrEP'.

The majority of respondents $(\mathrm{n}=39$ of $42,92.8 \%$ ) had never consulted patients regarding PrEP, with more than half of participating GPs ( $\mathrm{n}=30$ of $42,71.4 \%)$ responding 'not at all' when asked how confident they felt about prescribing HIV PrEP.

The correct method of PrEP use was correctly identified by $24.4 \%(n=11$ of $45)$, while $37.8 \%(n=17$ of 43$)$ correctly identified the degree of effectiveness in preventing HIV. Of the 43 GPs who responded whether PrEP education should be part of HIV prevention discussions, $74.4 \%(n=32)$ either agreed $(n=18)$ or strongly agreed $(n=14)$, while only $4.4 \%$ $(n=2)$ disagreed or strongly disagreed that a consultation regarding PrEP should include discussion of other methods of HIV prevention. There was an association between younger age of GP and accurate knowledge of the correct method of PrEP prescription (medications and duration of treatment), which tended towards significance $(P=0.1)$.

Lack of knowledge was identified by the qualitative interviews as the GPs' primary reason for hesitation to prescribe PrEP. This topic was investigated by asking GPs to describe PrEP in their own words. Although most GPs were able to describe the basic premise of PrEP, no GPs were able to identify the medication class, specific medications or dosages used.

A minority of interviewed GPs had previously discussed PrEP with their patients. One had discussed PrEP with a patient on the QPrEP trial, and as such was not providing the patient any new information on PrEP, while another
GP had identified patients at risk and introduced the topic during their consult. Further discussion with this GP indicated that the consult concluded with a referral to a sexual health clinic.

Interviews highlighted an associated lack of confidence, not only in prescribing PrEP, but also engaging in discourse about PrEP. GPs were willing to outline the concept to patients, but were not confident to give in-depth information, as evidenced through: 'I feel like I could probably give them a basic outline ... but in terms of logistics and any other issues, I'd probably be uninformed' (GP5). GPs were hesitant when discussing concepts during interviews, but were willing to admit that their knowledge was 'pretty minimal' (GP8), which caused hesitancy towards PrEP (Table 1).

\section{High-risk populations}

GPs were asked to indicate which populations they would target with the prescription of PrEP. In total, $51.1 \%$ of the 45 GPs surveyed $(n=23)$ were very likely and $26.7 \%(n=12)$ likely to prescribe PrEP to serodiscordant (mixed HIV status) couples (Table 2). Almost half $(n=22)$ of the 45 GPs reported they were 'very likely' to prescribe PrEP to men who have sex with men (MSM) without condom use. Only $6.7 \%(n=3)$ of GPs indicated that they would be 'very likely' to prescribe PrEP to heterosexual patients who had multiple partners.

In terms of knowledge of appropriate populations for PrEP prescription, the majority of interview participants identified MSM as potentially benefiting the most from PrEP, with some minor discussion of the role it may play with serodiscordant couples. GPs identified MSM as an at-risk group. However, during the interviews, non-verbal cues from some participants, such as withdrawal of eye contact and hesitancy, indicated a sense of discomfort in discussing this particular group. There was some evidence of an undercurrent of discomfort to initiate conversation regarding PrEP, but rather a preference to allow the patients to start discussion, reinforced through the following quote: 'definitely them coming to me' (GP10). 
GPs indicated a strong desire for appropriate education, reinforcing the theme of lack of knowledge as an impediment to prescribing PrEP. There was consensus among those surveyed (Table 3) and interviewed that provision of education in the form of hard copies, guidelines and easily accessible information was beneficial to improve prescription and overcome barriers, 'because you've got to know where to find it' (GP4). It emerged that because GPs were unlikely to prescribe PrEP regularly, there was a concern that a single education session would not be sufficient to retain knowledge: 'unless you've got a stepwise in writing in front of me, I'm not going to remember how to do it. So I need written information' (GP2).

\section{Concerns}

GPs were most concerned about inexperience in prescribing antiretrovirals $(35.6 \%, \mathrm{n}=16$ of $45, \mathrm{CI}$ $95 \%$ very concerned) and the lack of guidelines for prescription, with $33.3 \%$ of GPs ( $n=15$ of 45$)$ indicating they were 'very concerned', and $31.1 \%(\mathrm{n}=14$ out of 45) 'concerned' (Table 4). GPs who correctly answered the PrEP knowledge component of the questionnaire were less likely to be concerned about the prescription of PrEP. Only $28.5 \%$ ( $n=2$ of 7) of GPs who correctly answered both knowledge questions were concerned with the prescription of PrEP, while those who answered both incorrectly were more likely to be very concerned ( $\mathrm{n}=22$ out of $32, P=0.058)$. There was an association between lack of knowledge and concern about increasing risky behaviour, which tended towards significance $(P=0.058)$.

During the qualitative interviews, GPs were asked if they had any additional concerns regarding PrEP being made available in the community, for example increasing risky sexual behaviour. The majority of GPs did not identify this as an issue of concern for them. This was illustrated through responses such as, 'It's not going to increase their promiscuity, you'd just rather have them safe than not' (GP4) and, 'I don't think it will make them any more or less silly with risk-taking behaviour' (GP9). In addition, GPs stated that discussion of PrEP should be incorporated with safe sex counselling, as PrEP should not be their only form of protection. As such, the importance of adherence and counselling regarding PrEP was highlighted, emphasising the importance of additional protection: 'I wouldn't be as concerned about compliance as somebody who was using it as their whole and sole cause of protection' (GP9).

There was, overall, a sense of positivity and optimistic anticipation at potential population control of HIV. The majority of GPs were excited about the prospect of population control of HIV, as evidenced through: 'I think it's very exciting ... that you have a way to prevent the spread of HIV' (GP1). GPs were positive when discussing this theme during interviews, reinforcing the importance of creating safe environments and decreasing risk, as well as re-iterating the old adage: 'It's

\section{Table 1. Summary of qualitative themes and indicative quotes}

\begin{tabular}{lll}
\hline Theme & Subtheme & Selected narrative \\
\hline Positivity & $\begin{array}{l}\text { Optimism regarding population } \\
\text { control of HIV }\end{array}$ & 'I think it's awesome' (GP8) \\
& 'I see it as a positive thing. I think it makes people feel safer' (GP1) \\
\cline { 2 - 3 } & 'It makes sense from a population health point of view' (GP5) \\
\hline Barriers & '[PrEP should be seen] certainly as part of an STI screen' (GP9) \\
\hline
\end{tabular}

Subthemes of barriers

\begin{tabular}{|c|c|c|}
\hline Lack of knowledge & Lack of confidence & '... knowledge of how to prescribe it (is a concern)' (GP3) \\
\hline \multirow[t]{3}{*}{ Undesirable consequences } & Increased risky behavior & $\begin{array}{l}\text { 'I don't think it has any difference if they have PrEP on sexual } \\
\text { practices or not' (GP7) }\end{array}$ \\
\hline & \multirow[t]{2}{*}{ Not preferred population } & 'I don't want my practice full of those people'(GP2) \\
\hline & & $\begin{array}{l}\text { '... it's not really something I want to encourage ... men to have sex } \\
\text { with men' (GP2) }\end{array}$ \\
\hline
\end{tabular}

GP, general practitioner; HIV, human immunodeficiency virus; PrEP, pre-exposure prophylaxis; STI, sexually transmissible infection 
much better to prevent that infection ... than to go through all the treatment' (GP10). Optimism was evident regarding the mental health, as well as the public health, benefits of PrEP: 'I see it [PrEP] as a positive thing. I think it makes people feel safer' (GP1).

While a positive attitude to PrEP was reflected by the majority of GPs, a minor concern was expressed regarding consulting with 'that type of people' as 'it's not really something I really particularly want to encourage ... I'm not going be advertising it. I certainly don't want my practice full of people that want it' (GP2), suggesting more of a judgement or disapproval of the specific population involved, rather than PrEP itself. An additional judgement emerged in the form of, 'I'm not someone that, wants to promote promiscuity' (GP7) evidencing a fear that provision of PrEP would encourage such behaviour. However, the dominant theme was supportive towards PrEP, with additional positive points being 'prevention is always better than a cure' (GP3) and, 'this is about patients, not about me' (GP4) and, 'it makes sense from a population health point of view' (GP5).

\section{Discussion}

The aim of this study was to explore GP knowledge of and attitudes to PrEP. While GPs predominantly supported prescription of PrEP to appropriate populations, they expressed some concern regarding lack of knowledge and confidence to do so. As PrEP was listed on the PBS in April 2018 it is imperative that GPs be adequately informed and prepared to prescribe PrEP. It was also surprising that participating GPs did not indicate concern regarding the potential for an increase in risky sexual behaviour and increasing rates of sexually transmissible infections (STIs), as recent studies have shown evidence of an increase in high-risk sexual activity as well as increased prevalence of rectal chlamydia and gonorrhoea. ${ }^{11-14}$

Our study was conducted prior to the publication of the updated 2018
ASHM clinical guidelines for PrEP. ${ }^{21}$ Both our quantitative and qualitative analyses identified that the provision of national guidelines and education would be considered beneficial by GPs. GPs indicated that they would prefer hard copies of guidelines. Other studies have indicated that formalised guidelines on PrEP prescription from a recognised institution would influence GP prescribing. 6,9,10,22,23 The ASHM has since released a range of education options for PrEP providers including full guidelines, webinars, online learning modules and a two-page decision-making tool. ${ }^{21}$ It is important that education continues to be offered in a variety of modalities to reach all providers. ${ }^{24}$

As well as education about PrEP, it will be important to educate GPs on sexual history-taking and increasing STI screening, as current medical training in obtaining sexual histories and caring for lesbian, gay, bisexual, transgender, intersex, queer or questioning (LGBTIQ) people is suboptimal. ${ }^{13,25-27}$

\section{Table 2. Likelihood to prescribe pre-exposure prophylaxis, $\mathbf{n}(\%)$}

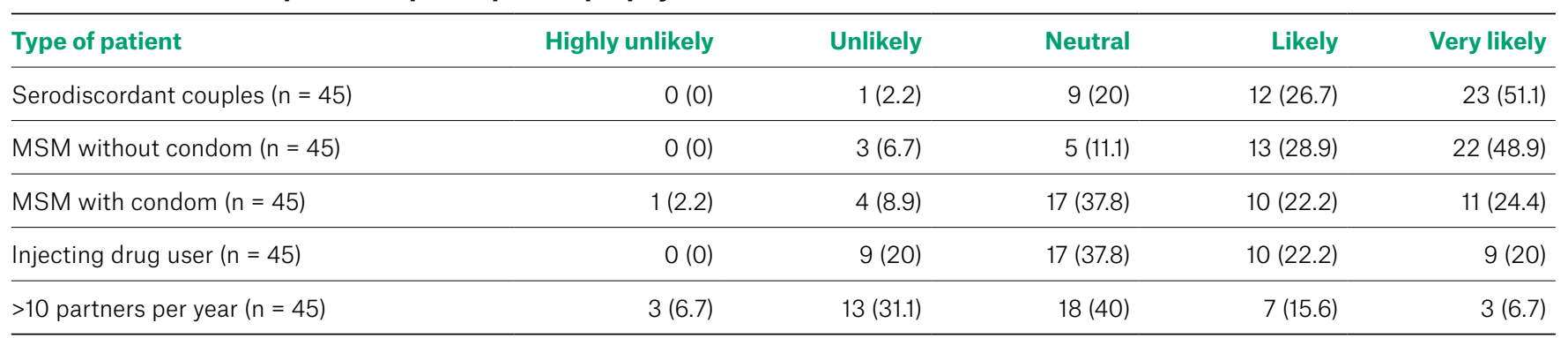

MSM, men who have sex with men

Table 3. Ways to increase confidence, $\mathbf{n}(\%)$

\begin{tabular}{lrrrrr}
\hline Method & $\begin{array}{r}\text { Not at all } \\
\text { important }\end{array}$ & $\begin{array}{r}\text { Low } \\
\text { importance }\end{array}$ & Neutral & $\begin{array}{r}\text { Moderately } \\
\text { important }\end{array}$ & $\begin{array}{r}\text { Very } \\
\text { important }\end{array}$ \\
\hline National guidelines $(n=45)$ & $3(6.7)$ & $1(2.2)$ & $2(4.4)$ & $15(33.3)$ & $27(60)$ \\
\hline Evidence of efficacy $(n=45)$ & $1(2.2)$ & $1(2.2)$ & $9(20)$ & $17(37.8)$ & $19(42.2)$ \\
\hline Cost information $(n=45)$ & $0(0)$ & $3(6.7)$ & $8(17.8)$ & $17(37.8)$ & $17(37.8)$ \\
\hline Cost analysis $(n=45)$ & $1(2.2)$ & $3(6.7)$ & $12(26.7)$ & $20(44.4)$ & $9(20)$ \\
\hline Foreign guidelines $(n=45)$ & $2(4.4)$ & $1(2.2)$ & $16(35.6)$ & $17(37.8)$ & $9(20)$ \\
\hline Evidence of patient acceptability $(n=45)$ & & & $17)$
\end{tabular}




\section{Table 4. Clinician concerns regarding pre-exposure prophylaxis, $\mathbf{n}(\%)$}

\begin{tabular}{|c|c|c|c|c|c|}
\hline Type of concern & $\begin{array}{r}\text { Not at all } \\
\text { concerned }\end{array}$ & $\begin{array}{r}\text { Slightly } \\
\text { concerned }\end{array}$ & Neutral & $\begin{array}{l}\text { Moderately } \\
\text { concerned }\end{array}$ & $\begin{array}{r}\text { Very } \\
\text { concerned }\end{array}$ \\
\hline Inexperience prescribing antiretroviral $(n=45)$ & $4(8.9)$ & $4(8.9)$ & $6(13.3)$ & $13(28.9)$ & $16(35.6)$ \\
\hline Prescription of $\operatorname{PrEP}(n=45)$ & $9(20)$ & $3(6.7)$ & $12(26.7)$ & $11(24.4)$ & $10(22.2)$ \\
\hline Resistance $(n=45)$ & $6(13.3)$ & $14(31.1)$ & $16(35.6)$ & $6(13.3)$ & $3(6.7)$ \\
\hline Lack of PrEP trials $(n=44)^{*}$ & $7(15.6)$ & $4(8.9)$ & $24(53.3)$ & $7(15.6)$ & $2(2.5)$ \\
\hline Cost $(n=45)$ & $9(15.6)$ & $4(8.9)$ & $21(46.7)$ & $3(6.7)$ & $5(11.1)$ \\
\hline
\end{tabular}

*One general practitioner did not respond to this question

PrEP; pre-exposure prophylaxis

It should be acknowledged that the analysis and generalisability of our findings is limited as we only sampled a small number of GPs in one regional centre in North Queensland. However our return rate of $41.1 \%$ (45 of 109) was higher than average for this type of study, and we sampled close to half of the GPs in the region.

\section{Recommendations}

In order for PrEP to be effective, it needs to be prescribed in general practice. PrEP is not just a medication - it should be a program of prevention including an education program encouraging condom use to decrease risk of transmission of other STIs.

With Mackay GPs showing positivity towards HIV PrEP, the future of PrEP is optimistic. As PrEP has recently been accepted on the Australian PBS, this is an opportune time to provide education sessions to further improve GPs' knowledge and subsequently increase appropriate prescription. As of April 2018, the ASHM has released clinical guidelines for the use of $\operatorname{PrEP} ;{ }^{18}$ we recommend the creation of hard copies and easy access links for GPs to improve use.

\section{Conclusion}

HIV PrEP was listed on the PBS in April 2018 , resulting in a reduction in cost and increased accessibility. GPs lack knowledge regarding PrEP. There is an attitude of positivity towards PrEP despite the GPs' lack of confidence and comfort in prescribing. Consequently, there is a place for education and distribution of guidelines to GPs to promote the future implementation of PrEP in general practice.

\section{Authors}

William Lane MBBS, student, James Cook University, Qld

Clare Heal PhD, MBChB, Promotional Chair, Discipline of General Practice and Rural Medicine, James Cook University, Qld

Jennifer Banks, PhD, Senior Research Support, James Cook University, Qld. jennifer.banks2@jcu.edu.au Competing interests: None.

Funding: None.

Provenance and peer review: Not commissioned, externally peer reviewed.

\section{References}

1. Spinner CD, Boesecke C, Zink A, et al. HIV preexposure prophylaxis (PrEP): A review of current knowledge of oral systemic HIV PrEP in humans. Infection 2016;44(2):151-58. doi: 10.1007/s15010015-0850-2.

2. Food and Drug Administration. Truvada for PrEP fact sheet: Ensuring safe and proper use. Silver Spring, MD: FDA, 2012

3. Department of Health: Therapeutic Goods Administration. Australian Public Assessment Report for tenofovir disoproxil fumarate/ emtricitabine. Woden, ACT: Therapeutic Goods Administration Australia, 2016.

4. Grant RM, Lama JR, Anderson PL, et al. Preexposure chemoprophylaxis for HIV prevention in men who have sex with men. N Engl J Med 2010;363(27):2587-99. doi: 10.1056/ NEJMoa1011205.
5. Bacon O, Gonzalez R, Andrew E, et al. Brief report: Informing strategies to build PrEP capacity among San Francisco bay area clinicians. J Acquir Immune Defic Syndr 2017;74(2):175-79. doi: 10.1097/QAI.0000000000001182.

6. Hakre S, Blaylock JM, Dawson P, et al. Knowledge, attitudes, and beliefs about HIV pre-exposure prophylaxis among US Air Force health care providers. Medicine (Baltimore) 2016;95(32):e4511. doi: 10.1097/MD.0000000000004511.

7. Mimiaga MJ, White JM, Krakower DS, Biello KB, Mayer KH. Suboptimal awareness and comprehension of published preexposure prophylaxis efficacy results among physicians in Massachusetts. AIDS Care 2014;26(6):684-93. doi: 10.1080/09540121.2013.845289.

8. Ross I, Mejia C, Melendez J, et al. Awareness and attitudes of pre-exposure prophylaxis for HIV prevention among physicians in Guatemala: Implications for country-wide implementation. PLoS One 2017;12(3):e0173057. doi: 10.1371/ journal.pone.0173057.

9. Smith DK, Mendoza MC, Stryker JE, Rose CE. PrEP awareness and attitudes in a national survey of primary care clinicians in the United States, 2009-2015. PLoS ONE 2016;11(6):e0156592. doi: 10.1371/journal.pone.0156592.

10. White JM, Mimiaga MJ, Krakower DS, Mayer KH. Evolution of Massachusetts physician attitudes, knowledge, and experience regarding the use of antiretrovirals for HIV prevention. AIDS Patient Care STDS 2012;26(7):395-405. doi: 10.1089/ apc.2012.0030.

11. Traeger MW, Schroeder SE, Wright EJ, et al. Effects of pre-exposure prophylaxis for the prevention of HIV infection on sexual risk behavior in men who have sex with men: A systematic review and meta-analysis. Clin Infect Dis 2018;67(5):676-86. doi: 10.1093/cid/ciy182.

12. Beymer MR, DeVost MA, Weiss RE, et al. Does HIV pre-exposure prophylaxis use lead to a higher incidence of sexually transmitted infections? A case-crossover study of men who have sex with men in Los Angeles, California. Sex Transm Infect 2018;94(6)457-62. doi: 10.1136/ sextrans-2017-053377. 
13. Buchbinder SP. Maximizing the benefits of HIV preexposure prophylaxis. Top Antivir Med 2018;25(4):138-42.

14. Traeger MW, Cornelisse VJ, Asselin J, et al. Association of HIV preexposure prophylaxis with incidence of sexually transmitted infections among individuals at high risk of HIV infection. JAMA 2019;321(14):1380-90. doi: 10.1001/ jama.2019.2947.

15. Lurie P, Miller S, Hecht F, Chesney M, Lo B. Postexposure prophylaxis after nonoccupational HIV exposure: Clinical, ethical, and policy considerations. JAMA 1998;280(20):1769-73. doi: 10.1001/jama.280.20.1769.

16. Cornelisse V. PrEP on the PBS: An opportunity in HIV prevention. Surrey Hills, NSW: NPS Medicinewise, 2018. Available at www.nps.org. au/news/prep-on-the-pbs-an-opportunity-in-hivprevention [Accessed 3 July 2019].

17. Australasian Sexual Health and Medicine, Positive Life NSW, ACON. PrEP access options. NSW: ACON, 2017. Available at www.endinghiv.org.au/ wp-content/uploads/2017/05/Ending-HIV-PrEPAccess-Options-UPDATE-2017.pdf [Accessed 30 July 2019].

18. Wright E, Grulich A, Roy K, et al. Australasian Society for HIV, Viral Hepatitis and Sexual Health Medicine HIV pre-exposure prophylaxis: Clinical guidelines. Update April 2018. J Virus Erad 2018;4(3):143-59.

19. Creswell J, Plano Clark VL. Designing and conducting mixed methods research. 2 nd edn. Sage: Thousand Oaks, 2011.

20. PHN Northern Queensland. PHN Northern Queensland: An Australian Government Initiative. Cairns, QId: NQPHN, 2016. Available at www.nqphn.com.au [Accessed 3 July 2019].

21. Australasian Society for HIV, Viral Hepatitis and Sexual Health Medicine [ASHM]. Update on PrEP for HIV clinicians, Australia 2019. Sydney: ASHM, 2019. Available at www.ashm.org.au/HIV/PrEP [Accessed 3 July 2019].
22. Arnold EA, Hazelton $P$, Lane T, et al. A qualitative study of provider thoughts on implementing pre-exposure prophylaxis (PrEP) in clinical settings to prevent HIV infection. PLoS ONE 2012;7(7):e40603. doi: 10.1371/journal. pone.0040603.

23. Krakower DS, Ware NC, Maloney KM, Wilson IB, Wong JB, Mayer KH. Differing experiences with pre-exposure prophylaxis in Boston among lesbian, gay, bisexual, and transgender specialists and generalists in primary care: Implications for scale-up. AIDS Patient Care STDS 2017;31(7):297-304. doi: 10.1089/apc.2017.0031.

24. Terndrup C, Streed CG Jr, Tiberio P, et al. A cross sectional survey of internal medicine resident knowledge, attitudes, behaviors, and experiences regarding pre-exposure prophylaxis for HIV infection. J Gen Intern Med 2019. doi: 10.1007/ s11606-019-04947-2.

25. Loeb DF, Lee RS, Binswanger IA, Ellison MC, Aagaard EM. Patient, resident physician, and visit factors associated with documentation of sexual history in the outpatient setting. J Gen Intern Med 2011;26(8):887-93. doi: 10.1007/s11606-011-1711-z.

26. Obedin-Maliver J, Goldsmith ES, Stewart L, et al. Lesbian, gay, bisexual, and transgender-related content in undergraduate medical education. JAMA 2011;306(9):971-77.

27. Mayer KH, Bekker L-G, Stall R, Grulich AE Colfax G, Lama JR. Comprehensive clinical care for men who have sex with men: An integrated approach. Lancet 2012;380(9839):378-87. doi: 10.1016/S0140-6736(12)60835-6. 